\title{
Defective composite restorations - repair or replace?
}

\section{Abstracted from}

Sharif Mo, Catleugh M, Merry A et al.

Replacement versus repair of defective restorations in adults: resin composite. Cochrane Database Syst Rev 2014; 2: Art. No.: CD005971. DOI: 10.1002/14651858.CD005971.pub3

Address for correspondence: Cochrane Oral Health Group, School of Dentistry, The University of Manchester, Coupland 3 Building, Oxford Road, Manchester, UK. M13 9PL E-mail: cohg@manchester.ac.uk

\section{Question: Should we repair or replace defective composite restorations?}

Data sources The Cochrane Oral Health Group's Trials Register, the Cochrane Central Register of Controlled Trials (CENTRAL), Medline, Embase, BIOSIS via Web of Knowledge, Web of Science and Opengrey databases were searched. In addition researchers and experts in the field were contacted to trace unpublished or ongoing studies. No restrictions were placed on the language or date of publication.

Study selection Randomised controlled trials (including split-mouth studies), involving replacement and repair of resin composite restorations in adults with a defective molar restoration in a permanent molar or premolar teeth were to be considered.

Data extraction and synthesis Two review authors independently assessed titles and abstracts for each article identified by the searches in order to decide whether the article was likely to be relevant. Full papers were obtained for relevant articles and both review authors studied these. The Cochrane Collaboration statistical guidelines were followed for data synthesis.

Results The search strategy retrieved 298 potentially eligible studies, after de-duplication. After examination of the titles and abstracts, full texts of potentially relevant studies were retrieved, but none of the retrieved studies met the inclusion criteria of the review.

Conclusions There are no published randomised controlled trials relevant to this review question. Therefore there is a need for methodologically sound randomised controlled trials that are reported according to the Consolidated Standards of Reporting Trials (CONSORT) statement (www.consort-statement.org/). Further research also needs to explore qualitatively the views of patients on repairing versus replacement, and investigate themes around pain, anxiety and distress, time and costs.

This paper is based on a Cochrane Review published in the Cochrane Library 2014, issue 2 (see www.thecochranelibrary.com for information). Cochrane Reviews are regularly updated as new evidence emerges and in response to feedback, and the Cochrane Library should be consulted for the most recent version of the review.

\section{Commentary}

The replacement or repairing of dental restorations is one of the most common procedures performed by general dentists. ${ }^{1,2}$ These procedures are necessitated by marginal breakdown, restoration failure and/or recurrent caries.

With the increase in frequency of placing composite restorations in posterior teeth and the emphasis on conserv tive management in restorative care, the review authors attempted to address the issue of repair versus replacement of posterior composites.

There were many strengths regarding the review methodology. As the clinical question involved the effectiveness of two different therapeutic interventions, only randomised controlled trials on repair or replacement of defective composite restorations in adult premolars or molars were eligible for inclusion.

The primary and secondary outcomes were clearly described with an emphasis on patient-oriented outcomes. Multiple electronic databases, without language or publication status, with targeted search strategies were used, as well as hand-searching of appropriate journals for studies potentially meeting the inclusion criteria.

Two review authors independently assessed the abstracts and a thorough assessment of bias was planned for studies meeting the inclusion criteria. Having no studies meet the inclusion criteria for this review does not mean this systematic review has no value. Rather, it points out gaps in our knowledge and understanding of composite restoration repair and areas where further study is needed. There have been some observational studies published on this topic. A recent survey of US dental schools has shown that a majority teach composite restoration repair both didactically and clinically. ${ }^{3}$

A recently published cross-sectional study from a dental practice based research network showed that $75 \%$ of all posterior restorations were replaced and only $25 \%$ were repaired. ${ }^{1}$ Factors associated with restoration repair were more recent dental graduates, restorations other than amalgam and older patients. Additionally, composite restorations have higher repair rates as compared with amalgam in both the primary and permanent dentitions. ${ }^{4}$

Yet, restoration repair has shown promise as a recent cohort study showed that repair can increase restoration longevity as it can minimise tooth reduction associated with replacement. ${ }^{2}$

Evidence-based clinical decision-making requires practitioners 
to use the highest levels of evidence. In the absence of randomised trials, observational studies can provide guidance for clinicians in an effort to improve treatment outcomes.

Elliot Abt

Deptartment of Dentistry, Illinois Masonic Medical Center, Chicago, IL, USA

1. Gordon VV, Riley JL, Geraldeli S, et al. Repair or replacement of defective restorations by dentists in The Dental Practice-Based Research Network. / Am Dent Assoc 2012; 143: $593-601$.

2. Fernandez EM, Martin IA, Angel PA, Mjör IA, Gordan VV, Moncada GA. Survival rate of sealed, refurbished and repaired defective restorations: 4-year follow-up. Braz Dent / 2011; 22: 134-139.

3. Lynch CD, Blum IR, Frazier KB, Haisch LD, Wilson NH. Repair or replacement of defective direct resin-based composite restorations: contemporary teaching in US and Canadian dental schools. I Am Dent Assoc 2012; 143: 157-163.

4. Cacho MR. Composite restorations have a higher frequency of repair compared to amalgam restorations when place in posterior permanent teeth in pediatric patients (2011) CAT no. 768: UTHSCA Dental School CAT Library.
Evidence-Based Dentistry (2014), 15, 52-53. doi: 10.1038/sj.ebd.6401027

\section{Responding to commentaries in this journal}

If you wish to comment on any issues raised by this commentary or any others in the journal pleased direct your correspondence to:

Editor,

Evidence-based Dentistry Journal,

Nature Publising Group,

The Macmillan Building,

4 Crinan Street,

London N1 9XW

Email: ebdeditor@nature.com

as the address for correspondence given at the beginning of each commentary relates to the details of the author/s of the original paper upon which the content presented is based. 